\title{
Intravenous thrombolysis and platelet count
}

Neurology ${ }^{\circledR}$ 2018;91:852. doi:10.1212/WNL.0000000000005498

In the article "Intravenous thrombolysis and platelet count" by Gensicke et al., ${ }^{1}$ there were a few slight errors in table 3 published ahead of print on January 24, 2018. The corrected table was published online on February 20, 2018, and is included below with corrections in bold type. $p$ Values did not change. The authors regret the errors.

Table 3 Multivariable analysis of outcomes (odds adjusted for variables with $p<0.1$ in the univariable analysis): OR (95\% Cl) and $p$ value

\begin{tabular}{|c|c|c|c|}
\hline \multirow{2}{*}{$\begin{array}{l}\text { Putative predicting } \\
\text { variables }\end{array}$} & \multicolumn{3}{|c|}{ Outcome measures } \\
\hline & sICH & Poor outcome & Mortality \\
\hline Low $\mathrm{PC}^{\mathrm{b}}$ vs normal PC & $1.73(1.24-2.43) p=0.002^{d}$ & $0.89(0.39-1.97) p=0.499^{\mathrm{e}}$ & $1.09(0.83-1.44) p=0.615^{f}$ \\
\hline $\begin{array}{l}\mathrm{PC}<100 \times 10^{9} / \mathrm{L} \text { vS } \\
\mathrm{PC} \geq 100 \times 10^{9} / \mathrm{L}\end{array}$ & $1.56(0.48-5.07) p=0.433^{g}$ & $1.63(0.82-3.24) p=0.163^{h}$ & $1.38(0.64-2.98) p=0.368^{g}$ \\
\hline High PC' vs normal PC & $0.63(0.20-1.99) p=0.427^{g}$ & $1.51(0.96-2.38) p=0.074^{\mathrm{j}}$ & $2.02(1.21-3.37) p=0.006^{\mathrm{h}}$ \\
\hline $\begin{array}{l}\text { Decreasing PC } \\
\left.\text { (by } 10 \times 10^{9} / \mathrm{L}\right)\end{array}$ & $1.03(1.02-1.05) p<0.001^{d}$ & $0.99(0.98-0.99) p<0.001^{\mathrm{k}}$ & $0.98(0.98-0.99) p=0.001^{k}$ \\
\hline
\end{tabular}

Abbreviations: $\mathrm{Cl}=$ confidence interval; $\mathrm{OR}=$ odds ratio; $\mathrm{PC}=$ platelet count. $\mathrm{sICH}=$ symptomatic intracerebral hemorrhage (Second European-Australasian Acute Stroke Study [ECASS] definition).

a Poor outcome: modified Rankin Scale score of 3 to 6 . Final model of backward regression adjusted for other independent predictors.

b Low PC < $150 \times 10 \%$ L.

${ }^{\mathrm{C}}$ Normal PC $=150$ to $450 \times 10^{9} / \mathrm{L}$.

${ }^{\mathrm{d}}$ Age, stroke severity (NIH Stroke Scale score), glucose.

e Age, stroke severity (NIH Stroke Scale score), estimated glomerular filtration rate, glucose, current smoking, prior ischemic stroke.

${ }^{f}$ Age, sex, stroke severity (NIH Stroke Scale score), glucose, prior use of any antithrombotic, coronary artery disease.

${ }^{g}$ No adjustment because of the small number of outcome events.

h Stroke severity (NIH Stroke Scale score).

' High PC $>450 \times 10^{9} / \mathrm{L}$ platelet.

j Age, sex, stroke severity (NIH Stroke Scale score).

${ }^{k}$ Age, stroke severity (NIH Stroke Scale score), glucose, estimated glomerular filtration rate.

\section{Reference}

1. Gensicke H, Al Sultan AS, Strbian D, et al. Intravenous thrombolysis and platelet count. Neurology 2018;90:e690-e697. 


\section{Neurology}

\section{Intravenous thrombolysis and platelet count \\ Neurology 2018;91;852 \\ DOI 10.1212/WNL.0000000000005498}

This information is current as of October 29, 2018

Updated Information \& Services

References

Permissions \& Licensing

Reprints including high resolution figures, can be found at: http://n.neurology.org/content/91/18/852.full

This article cites 1 articles, 1 of which you can access for free at: http://n.neurology.org/content/91/18/852.full\#ref-list-1

Information about reproducing this article in parts (figures,tables) or in its entirety can be found online at:

http://www.neurology.org/about/about_the_journal\#permissions

Information about ordering reprints can be found online:

http://n.neurology.org/subscribers/advertise

Neurology ${ }^{\circledR}$ is the official journal of the American Academy of Neurology. Published continuously since 1951, it is now a weekly with 48 issues per year. Copyright (O 2018 American Academy of Neurology. All rights reserved. Print ISSN: 0028-3878. Online ISSN: 1526-632X.

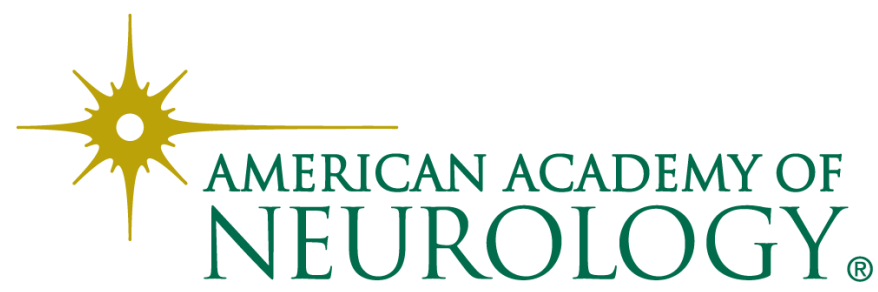

